\title{
PET/CT Imaging and Human Papilloma Virus-Positive Oropharyngeal Squamous Cell Cancer: Evolving Clinical Imaging Paradigm
}

\author{
Rathan M. Subramaniam ${ }^{1-3}$, Krishna C. Alluri ${ }^{1}$, Abdel K. Tahari ${ }^{1}$, Nafi Aygun ${ }^{1}$, and Harry Quon ${ }^{2,4,5}$ \\ ${ }^{I}$ Russell H. Morgan Department of Radiology and Radiological Sciences, Johns Hopkins School of Medicine, Baltimore, Maryland; \\ ${ }^{2}$ Department of Otolaryngology-Head and Neck Surgery, Johns Hopkins School of Medicine, Baltimore, Maryland; ${ }^{3}$ Department of \\ Health Policy and Management, Johns Hopkins Bloomberg School of Public Health, Baltimore, Maryland; ${ }^{4}$ Department of Radiation \\ Oncology and Molecular Radiation Sciences, Johns Hopkins School of Medicine, Baltimore, Maryland; and ${ }^{5}$ Medical Oncology, \\ Johns Hopkins School of Medicine, Baltimore, Maryland
}

\begin{abstract}
Learning Objectives: On successful completion of this activity, participants should be able to (1) understand the clinical pathologic features of human papilloma virus-positive oropharyngeal squamous cell cancer; (2) review the evolving role of ${ }^{18} \mathrm{~F}-\mathrm{FDG}$ PET/CT in the management of patients with human papilloma virus-positive oropharyngeal squamous cell cancer; and (3) postulate the imaging strategy of management of patients with human papilloma viruspositive oropharyngeal squamous cell cancer.
\end{abstract}

Financial Disclosure: Dr. Subramaniam is a speaker for Eli Lilly on amyloid brain imaging. The authors of this article have indicated no other relevant relationships that could be perceived as a real or apparent conflict of interest.

CME Credit: SNMMI is accredited by the Accreditation Council for Continuing Medical Education (ACCME) to sponsor continuing education for physicians. SNMMI designates each JNM continuing education article for a maximum of 2.0 AMA PRA Category 1 Credits. Physicians should claim only credit commensurate with the extent of their participation in the activity. For CE credit, participants can access this activity through the SNMMI Web site (http:// www.snmmi.org/ce_online) through March 2017.

Human papillomavirus (HPV)-positive oropharyngeal squamous cell carcinoma (OPSCC) represents an emerging disease that differs from HPV-negative OPSCC in natural history and prognosis. Contrastenhanced PET/CT is essential to accurately stage the primary site when there are smaller tumors; neck nodal metastases, which tend to have a more cystic component; and distant metastases that manifest in unusual sites (disseminating phenotype) such as bones and other solid organs, including brain. Metastases tend to appear later in the disease course during follow-up for HPV-positive OPSCC than for HPV-negative OPSCC. Because HPV-positive OPSCC patients have a better clinical outcome, there is a need for treatment deintensification to spare the patient from treatmentrelated toxicities. ${ }^{18} \mathrm{~F}-\mathrm{FDG} \mathrm{PET} / \mathrm{CT}$ would play a role in monitoring patients with deintensified treatments to ensure that no adverse outcome is introduced. The better prognosis and outcome of HPV-positive OPSCC patients would warrant imaging follow-up that is less intense but continues longer because of the manifestation of distant metastases later in the disease course and at unusual sites. All these clinical paradigms facilitate a definite role for PET/CT imaging in the management of HPV-positive OPSCC.

Key Words: general oncology; PET/CT; HPV; OPSCC

J Nucl Med 2014; 55:431-438

DOI: 10.2967/jnumed.113.125542

Received Apr. 29, 2013; revision accepted Nov. 1, 2013.

For correspondence or reprints contact: Rathan M. Subramaniam, Russell H. Morgan Department of Radiology and Radiology Science, Johns Hopkins Medical Institutions, 601 N. Caroline St./JHOC 3235, Baltimore, MD 21287. E-mail: rsubram4@jhmi.edu

Published online Jan. 9, 2014

COPYRIGHT (C) 2014 by the Society of Nuclear Medicine and Molecular Imaging, Inc.
$\mathbf{M}$ cancers (HNSCC) (1). Tobacco and alcohol have been the major risk factors for development of HNSCC. However, the human papillomavirus (HPV) has been recognized as a major etiologic factor for a subset of HNSCCs arising from the oropharynx $(2,3)$ (HPV-positive oropharyngeal squamous cell carcinoma [OPSCC]) since the early 2000s, especially in the tongue base and palatine and lingual tonsils. Greater than $90 \%$ of HPV-positive OPSCCs are associated with a single HPV type, HPV-16 (4). HPV-positive OPSCCs are epidemiologically distinct from HPV-negative OPSCCs. HPV-positive OPSCC is characterized by younger age (usually 40-50 y) at onset, predominance in white men, and a strong association with sexual behaviors $(3,5,6)$.

PET/CT is a useful imaging test in staging, therapy response assessment, and follow-up of patients with many human solid tumors (7-12), including head and neck cancers (13-17). The objective of this article is to review the evolving role of PET/ $\mathrm{CT}$ in staging, therapy response assessment, and follow-up of patients with HPV-positive OPSCC.

\section{CLINICOPATHOLOGIC FEATURES}

\section{HPV Status}

The best method to detect the HPV status of the tumor is controversial, and both in situ hybridization and polymerase chain reaction are commonly used. Immunohistochemistry for p16 protein, a cyclin-dependent kinase inhibitor, could serve as a potential surrogate marker (4). In HPV-positive tumors, transcription of the viral oncoprotein E7 inactivates retinoblastoma protein, which leads to upregulation of p16 to levels that can be detected by immunohistochemistry. Many studies have demonstrated the correlation between HPV and p16 expression status in HNSCC (18-24). p16 immunohistochemistry is easier to interpret than HPV in situ hybrid- 
ization (25), as well as less expensive. However, the expression of p16 is not limited to HPV-positive tumors, and using this marker alone as an indicator of biologically relevant HPV infections inevitably entails the risk of including some false-positive results $(22,25)$. Detection of p16 can also indicate disruption of the retinoblastoma protein pathway by other causes, and HPV infection other than HPV16 type (24) can also lead to false-positives. In a study by Thomas and Primeaux (25), among the 47 HNSCC cases, 17 and 29 cases were positive with HPV in situ hybridization and p16 immunohistochemistry, respectively. p16 immunohistochemistry has been shown to have a sensitivity of $100 \%$ and a specificity of $79 \%$ in screening for transcriptionally active HPV infection. In a study by Pannone et al. (22), p16 immunohistochemistry showed a sensitivity of $100 \%$ and a specificity of $93 \%$ in the detection of HPV in 22 OPSCC cases. For clinical purposes, many centers perform p16 immunohistochemistry for detection of HPV-positive HNSCC, as there is more than $90 \%$ concordance between p16 expression by immunohistochemistry and in situ hybridization (24).

\section{Histology}

HPV-positive OPSCCs are histologically distinct from HPVnegative tumors. In contrast to the HPV-negative OPSCCs, which are usually moderately differentiated and keratinizing, HPV-positive OPSCCs are consistently poorly differentiated, demonstrate a high mitotic rate, are nonkeratinizing, and have a distinct basaloid appearance (26-29). There is repression of viral oncogene expression in HPV-positive OPSCC cells, which induce massive apoptosis and restoration of p53 and retinoblastoma protein tumor suppressor pathways. The high mitotic rate, apoptosis, and restoration of tumor suppressor pathways may explain the better survival prognosis in patients with HPV-positive OPSCC.

\section{Survival Benefit}

HPV-positive OPSCCs are reported to have improved overall and progression-free survival in comparison to their HPV-negative counterparts. In a study by Ang et al. (30), 63.8\% of patients with OPSCCs (206/323) had HPV-positive tumors. The authors retrospectively analyzed the association between tumor HPV status and survival among the patients with stage III or IV OPSCC. They found that patients with HPV-positive tumors had better 3-y rates of overall survival $(82.4 \%$ vs. $57.1 \%, P<0.001)$ than patients with HPV-negative tumors. HPV-positive patients had a $58 \%$ reduction in the risk of death (hazard ratio [HR], 0.42; 95\% confidence interval $[\mathrm{CI}], 0.27-0.66$ ), after adjustment for age, race, tumor and nodal stage, tobacco exposure, and treatment assignment.

In a metaanalysis by O'Rorke et al. (31) including 42 studies, the authors examined the survival differences between HPV-positive and HPV-negative HNSCC patients and found that patients with HPV-positive HNSCC had a $54 \%$ better overall survival than HPVnegative patients, with an HR of 0.46 (95\% CI, 0.37-0.57). The authors performed further subset analysis for OPSCC, and the pooled HR for overall survival was 0.47 (95\% CI, 0.35-0.62). The pooled HR for disease-specific survival was 0.28 (95\% CI, 0.19-0.40); similar effect sizes were found irrespective of the adjustment for confounders, HPV detection methods, or study location. Both progression-free survival and disease-free survival were significantly improved in HPV-positive HNSCCs. HPVpositive HNSCC patients and a subgroup of OPSCC patients had a significantly lower disease-specific mortality and were less likely to experience progression or recurrence of their cancer than were HPV-negative patients, in this metaanalysis.

\section{ROLE OF ${ }^{18}$ F-FDG PET/CT}

\section{Contrast-Enhanced PET/CT Protocol}

With experience and improvement in the technology, PET/CT can now be used to acquire a high-quality, integrated diagnostic CT scan for surgical planning along with the PET scan for metabolic imaging (Fig. 1). The advantage is to perform a comprehensive evaluation of patients with OPSCC in a single visit to the imaging department, improving workflow and convenience to patients and enhancing the integration of structural and functional information for clinicians. In general, nodal status and distant metastases are best evaluated by the PET component of contrast-enhanced PET/CT, and the morphology of the primary lesion as it relates to adjacent critical anatomic structures is best evaluated by the CT component.

${ }^{18}$ F-FDG PET/CT can be performed either with or without intravenous contrast material for the CT component. Our institutional practice is to have a dedicated head and neck PET acquisition and intravenous contrast administration for the CT component, according to the following protocol. Dedicated PET/ CT for the neck is performed from the skull base to the arch of the aorta with arms down and $120 \mathrm{~mL}$ of nonionic intravenous contrast material. The contrast agent is infused at a rate of $2 \mathrm{~mL} / \mathrm{s}$, and the scan is acquired $60 \mathrm{~s}$ after the infusion (automatic built-in delay). The CT parameters include $120 \mathrm{kVp}$, an intensity-modulated amperage (range, 30-400 mA), a pitch of 0.984 , and a slice thickness of $1.25 \mathrm{~mm}$. The second part of the study covers the clavicle to the mid thigh and is performed with arms up. The CT parameters include $120 \mathrm{kVp}$, an intensity-modulated amperage (range, 20-200 mA), a pitch of 0.984 , and a slice thickness of $3.75 \mathrm{~mm}$. PET is performed, again in 2 parts, with a $128 \times 128$

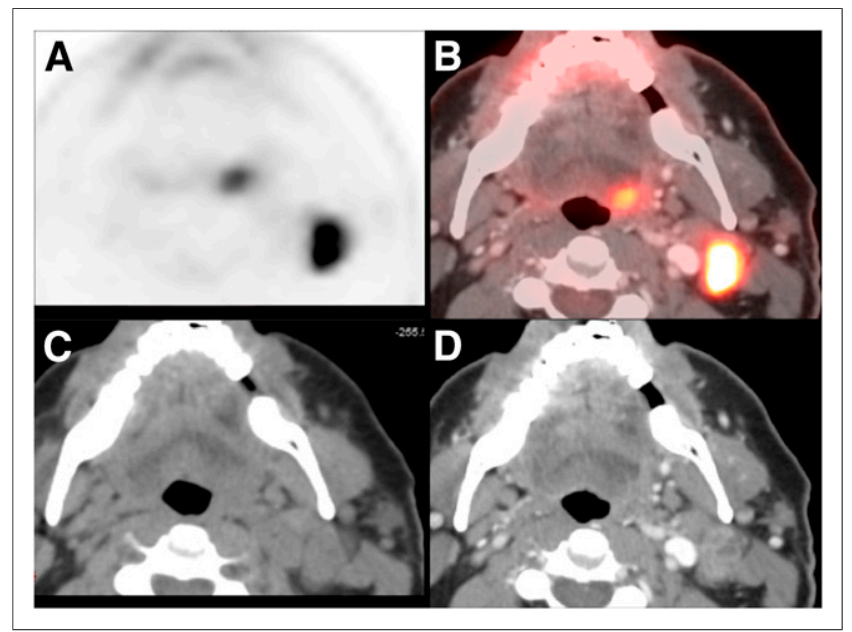

FIGURE 1. Small primary in 56-y-old man with history of T1 N2a MO left tongue base/tonsillar squamous cell cancer treated with definitive chemoradiation. Treatment initially was with cisplatin and later was switched to carboplatin for renal failure (intensity-modulated radiotherapy, 70 Gy), with excellent response. Primary site had SUV $\mathrm{max}_{\text {ax }}$ of 13.43, SUV $_{\text {peak }}$ of 9.52 , MTV of $18.39 \mathrm{~cm}^{3}$, and total lesion glycolysis of 105.85 g. Shown are axial PET (A), axial PET/CT (B), nonenhanced low-dose CT (C), and intravenous contrast-enhanced CT (D) images. Necrotic component of left IIA lymph node is nicely demonstrated by enhanced CT portion of PET/CT scan. Smaller primary lesion is more easily identifiable in PET portion of PET/CT scan. MTV is ${ }^{18} \mathrm{~F}-\mathrm{FDG}$-avid tumor volume segmented using gradient method. Total lesion glycolysis is MTV multiplied by SUV mean $_{\text {. }}$ 
matrix, 45-cm bed positions, a $30-\mathrm{cm}^{3}$ field of view for the head and neck, a $60-\mathrm{cm}$ field of view for the body, and $4.25 \mathrm{~min}$ for each bed position. The radiation dose from a nonenhanced ${ }^{18} \mathrm{~F}-\mathrm{FDG}$ PET/CT scan can vary between 8 and $30 \mathrm{mSv}$, depending on the protocol used, and usually is about $12-15 \mathrm{mSv}$ (32-34), and the radiation dose from contrast-enhanced neck CT is about $6-10 \mathrm{mSv}$.

\section{Evaluation of the Primary Site}

HPV-positive SCC arises predominantly from the lymphoepithelium of the oral cavity and oropharynx, including both palatine tonsils, the lingual tonsil, and the base of the tongue (35). The incidence of HPV-positive OPSCC increased at about $7.5 \%$ per year from 1998 to 2004, and therefore the percentage of OPSCC that was HPV-positive went from less than $20 \%$ to more than $70 \%$, likely due to sexual practices. HPV infection is responsible for $40 \%-80 \%$ of OPSCC (4). In a study by Gillison et al., 32 of 34 HPV-positive OPSCCs arose from the palatine and lingual tonsils, showing the distinct location of HPV-positive SCC (27). Fakhry et al. also showed that HPV-positive tumors were more likely than HPV-negative tumors to arise from the tonsil or base of the tongue $(P<0.001)(29)$.

HPV-positive OPSCCs are commonly occult (35). Deep within tonsillar crypts or hidden amid lingual tonsil fronds and mounds, early mucosal alterations due to HPV malignant transformation may grow undetected for many months. HPV-positive OPSCCs often present with smaller primary lesions (Fig. 1) than the HPV-negative OPSCCs, likely related to bulky neck nodal disease and earlier detection. In a study by Fakhry et al. (29), a greater number of HPV-positive than of HPV-negative OPSCCs were in the earlier T category (T2: $58 \%$ vs. $33 \%$, T3-T4: $42 \%$ vs. $67 \%$; $P=0.02)$. Ang et al. (30) also showed that HPV-positive OPSCCs were on average smaller at diagnosis (T2: $34.5 \%$ vs. $23.9 \%$, T3: $40.8 \%$ vs. $36.8 \%$, T4: $24.8 \%$ vs. $39.3 \% ; P=0.006)$ than were HPV-negative tumors.

Contrast-enhanced neck CT provides more anatomic details about the primary tumor, involvement of adjacent structures or muscles, and vascular anatomy than does the attenuation-correction CT done routinely in PET/CT. When enhanced CT and PET are combined as a single study, the study provides more accurate anatomic details along with the biologic functional information about the primary tumor (36). Krabbe et al. (37) conducted a retrospective study of whole-body ${ }^{18} \mathrm{~F}$-FDG PET/enhanced CT as a 1-step examination in the initial staging of unselected oral SCC and OPSCC. Seventy-three patients were included in the study, with 20 patients having OPSCC. ${ }^{18}$ F-FDG PET/enhanced CT results were correlated to histologic specimens obtained from tumor resection and neck dissection. ${ }^{18} \mathrm{~F}-\mathrm{FDG}$ PET/enhanced CT showed an overall sensitivity of $96 \%$ in detecting the primary tumor and a sensitivity of $100 \%$ among patients with OPSCC.

\section{Evaluation of Locoregional Nodal Metastases}

HPV-positive OPSCC patients frequently present with enlarged neck lymph nodes (Fig. 2). Cervical nodal metastases from HPVpositive OPSCC primary disease are usually in neck nodal levels II-IV. Contralateral nodal metastases are also seen, usually in conjunction with ipsilateral metastases but also occurring as an isolated event (35). HPV-positive OPSCCs are often associated with cystic lymph node metastases (38) and have a higher rate of nodal involvement than do HPV-negative OPSCCs $(29,39)$.

Patients with HPV-positive OPSCCs present with more advanced $\mathrm{N}$-stage disease than patients with HPV-negative OPSCCs

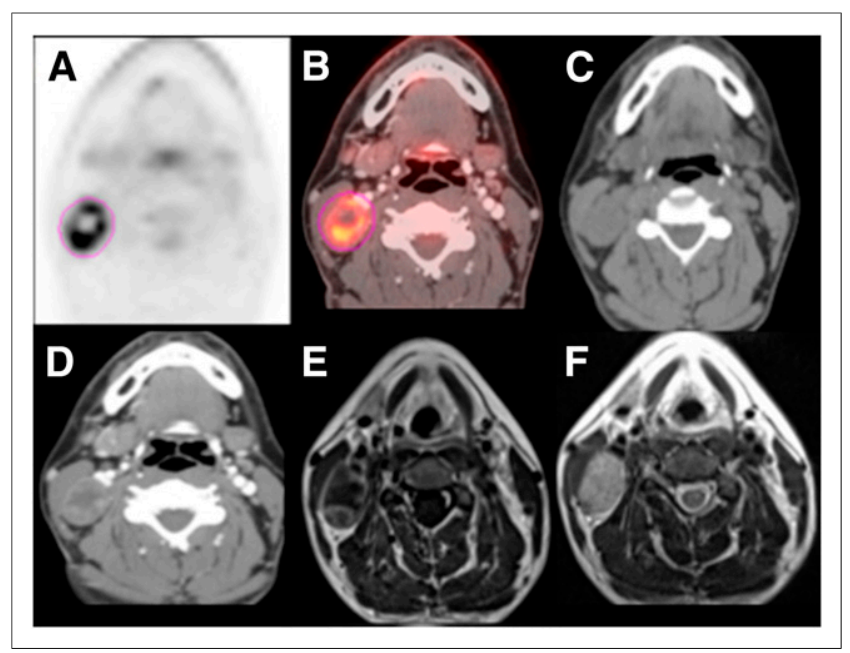

FIGURE 2. Cystic nodal metastasis and contrast-enhanced PET/CT in 29-y-old man with HPV-positive T4a N2b M0 squamous cell carcinoma of right lingual tonsil. Treatment was with definitive radiation and singleagent cisplatin to primary site and bilaterally to neck (total dose, 70 Gy in 200-cGy fractions). Axial PET (A) and fused PET/CT (B) demonstrate right level IIA neck nodal metastasis. Cystic or necrotic component of cervical nodal disease is not seen on nonenhanced CT (C) but can be appreciated on enhanced CT (D) and on enhanced T1-weighted (echo time/repetition time, 8.9/629 ms) (E) and T2-weighted (echo time/repetition time, 88/4,080 ms) (F) axial MR images. Right neck nodal metastasis had SUV $\max$ of 8.06, SUV $_{\text {peak }}$ of 6.12 , MTV of $18.40 \mathrm{~cm}^{3}$, and TLG of $62.36 \mathrm{~g}$.

$(29,39,40)$. Because the lymph nodes involved in HPV-positive OPSCCs often appear cystic, contrast-enhanced ${ }^{18} \mathrm{~F}-\mathrm{FDG}$ PET/ CT performs better than nonenhanced ${ }^{18} \mathrm{~F}-\mathrm{FDG}$ PET/CT in detecting cystic lymph node metastases (Fig. 2) (41). In a retrospective study by Haerle et al. (41), 34 patients with tonsillar SCC underwent pretreatment contrast-enhanced ${ }^{18} \mathrm{~F}$-FDG PET/CT followed by neck dissection as a standard of reference. The enhanced CT part, ${ }^{18} \mathrm{~F}$-FDG PET part, nonenhanced ${ }^{18} \mathrm{~F}$-FDG PET/CT part, and enhanced ${ }^{18} \mathrm{~F}$-FDG PET/CT part were assessed separately. The authors found that contrast-enhanced $\mathrm{CT}$ and contrast-enhanced ${ }^{18}$ F-FDG PET/CT perform equally well and that both perform better than nonenhanced ${ }^{18} \mathrm{~F}-\mathrm{FDG}$ PET/CT in detecting cystic lymph node metastases in tonsillar SCC. Therefore, in patients with tonsillar SCC scheduled for ${ }^{18} \mathrm{~F}$-FDG PET/CT, the authors recommended performing contrast-enhanced ${ }^{18} \mathrm{~F}-\mathrm{FDG}$ PET/CT.

\section{Evaluation of Distant Metastases}

There is a striking difference in the clinical behavior and prognosis of distant metastases between HPV-positive and HPVnegative OPSCC patients. Although the overall frequency of distant metastases is not related to HPV status, distant metastases among HPV-positive OPSCCs tend to disseminate to multiple organs and unusual sites (42). These metastases also can manifest later in the disease course, between 3 and $5 \mathrm{y}$ after completion of treatment $(42,43)$. In view of this characteristic pattern, the imaging of distant metastases during follow-up needs to be tailored.

To our knowledge, no published studies have specifically investigated the value of ${ }^{18} \mathrm{~F}-\mathrm{FDG}$ PET/CT in the diagnosis of distant metastases in a selected group of patients with HPV-positive OPSCC. However, as a whole-body examination, ${ }^{18}$ F-FDG PET/CT is especially helpful in the assessment of distant metastases in 


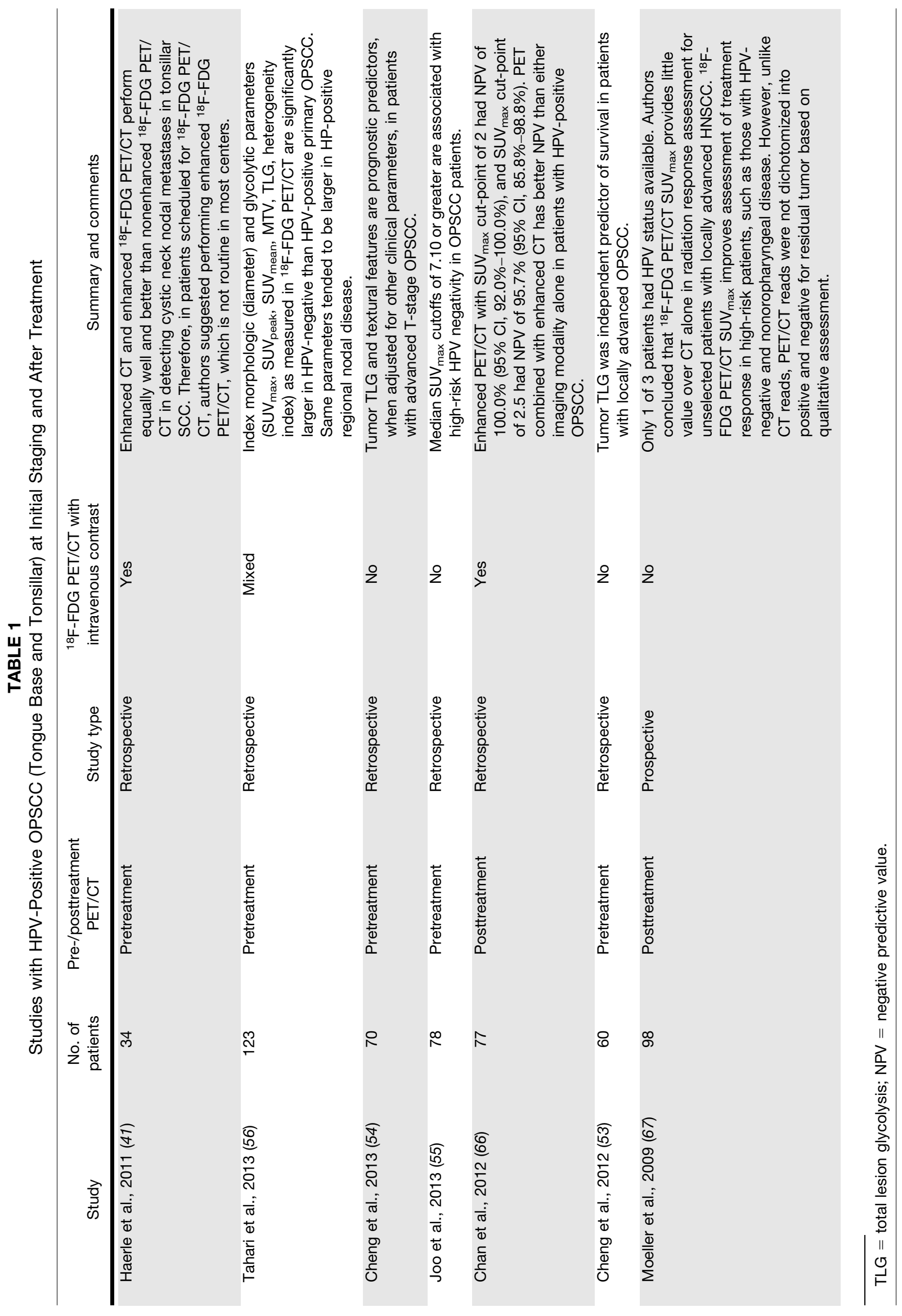

434 The Journal of Nuclear Medicine • Vol. 55 • No. 3 • March 2014 


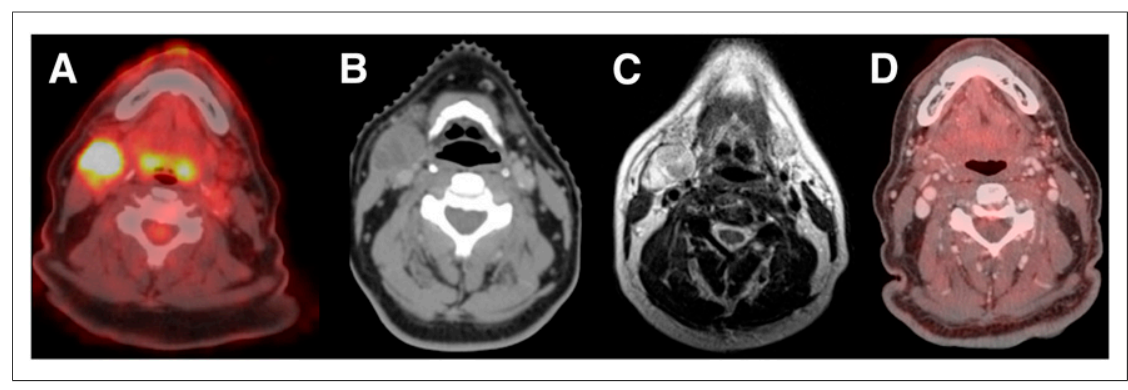

FIGURE 3. Complete metabolic response in 61-y-old man with history of HPV-positive T1 N2b M0 squamous cell carcinoma in right base of tongue. Treatment was with definitive concurrent radiation and cisplatin (to $70 \mathrm{~Gy}$ ). (A) Axial nonenhanced PET/CT shows mild asymmetry of ${ }^{18} \mathrm{~F}$ FDG uptake in right tongue base mass and intense ${ }^{18} \mathrm{~F}-\mathrm{FDG}$ uptake in right level lla nodal mass. ( $B$ and $C$ ) Cystic cervical nodal disease is better appreciated with enhanced $C T$ used for radiation treatment planning $(\mathrm{B})$ and enhanced T1-weighted MR imaging (echo time/repetition time, 8.9/577 ms) (C). (D) Follow-up enhanced PET/CT axial image demonstrates complete metabolic response to therapy, with residual scar tissue in right level II node.

HPV-positive OPSCC, particularly since they may manifest late, involve more than 2 organs, and appear at unusual sites.

A consistent trend toward higher sensitivity and diagnostic capability for PET/CT than for whole-body MR imaging has been shown in OPSCC and hypopharyngeal SCC $(44,45)$. A metaanalysis by $\mathrm{Xu}$ et al. (46) involving 15 studies with 1,445 patients with head and neck cancer showed pooled sensitivity and specificity estimates for ${ }^{18} \mathrm{~F}-\mathrm{FDG}$ PET/CT of 0.875 (95\% CI, 0.787-0.936) and 0.950 (95\% CI, 0.931-0.964), respectively, for detection of distant metastases. The superior accuracy of PET/CT for detection of distant metastases in head and neck cancer patients allows it to be deployed in the diagnosis of distant metastases in patients with HPV-positive OPSCC during the longer follow-up period that they require.

\section{Prognosis}

The high-dose chemotherapy and altered radiotherapy fractionation strategies used for treatment of OPSCC have improved survival rates. However, these strategies are also associated with an increased risk of developing late swallowing complications $(47,48)$. Studies have consistently demonstrated that HPV-positive OPSCC patients have better survival than HPV-negative OPSCC patients $(20,29-31,39,49)$. This difference in prognosis appears to be independent of the treatment modality used and suggests a fundamental difference in the biology of HPV-positive OPSCC. HPVpositive patients are typically younger, have fewer competing comorbidities, and, hence, are more likely to experience survivorship issues from current treatment approaches. With a diagnosis of head and neck cancer at a younger age and increased survival rates, the development of late swallowing complications becomes significant and is most likely to contribute to poor quality of life (50). To reduce treatment-related complications and improve quality of life for these patients, current research is evaluating deintensification of treatment to maintain the current survival rates while reducing longterm complications (such as swallowing dysfunction) (51). Such a possibility offers the potential to further improve patient selection at initial staging and to use PET/CT to assess the effectiveness of the deintensified treatment to avoid high treatment-failure.

Metabolic tumor volume (MTV) is the ${ }^{18} \mathrm{~F}-\mathrm{FDG}$-avid volume of the tumor or lesion. Many segmentation methods are available for calculation of MTV, the most common being use of a fixed percentage maximum standardized uptake value $\left(\mathrm{SUV}_{\max }\right)$ (such as $40 \%$ or $50 \%$ ), an absolute $\mathrm{SUV}_{\max }$ threshold (such as 2.5 or 3.0 or 5.0), and gradient segmentation (17). Tang et al. (52) prospectively studied the role of MTV obtained from pretreatment ${ }^{18} \mathrm{~F}$-FDG PET/CT in predicting treatment outcome in patients with head and neck cancer. The investigators also explored the interaction with p16 status as a surrogate marker for HPV. MTV and $\mathrm{SUV}_{\text {max }}$ were calculated for 83 patients with HNSCC who underwent ${ }^{18}$ F-FDG PET/CT before receiving definitive radiotherapy. The primary endpoint was to evaluate the relation between MTV and progression-free and overall survival. The investigators found that the primary tumor MTV predicted progression-free (HR, 1.94; $P<0.0001)$ and overall (HR, 1.57; $P<0.0001)$ survival, whereas nodal MTV did not. In the p16positive oropharynx subset $(n=64)$, total MTV remained a robust predictor of progression-free (HR, 4.23; $P<0.0001)$ and overall (HR, 3.21; $P=0.0029$ ) survival.

More studies have evaluated the characteristics and prognostic value of MTV, total lesion glycolysis, and HPV status in OPSCC at initial staging (Table 1) (53-56). Tahari et al. studied 123 newly diagnosed OPSCC patients (98 HPV-positive and $25 \mathrm{HPV}$-negative) who underwent ${ }^{18}$ F-FDG PET/CT at initial staging (56). HPV-negative primary tumors were significantly larger as measured by longest diameter $(P=0.002)$ and slightly more heterogeneous as measured by the ${ }^{18} \mathrm{~F}-\mathrm{FDG}$ heterogeneity index $(P=0.07)$ and had a higher $\operatorname{SUV}_{\text {max }}(\mathrm{P}=0.01), \operatorname{SUV}_{\text {peak }}(P=0.01), \mathrm{SUV}_{\text {mean }}$ $(P=0.01)$, MTV $(P=0.002)$, and total lesion glycolysis $(P=$ $0.001)$. There was no significant difference in the metabolic parameters of primary tumors or nodal metastases for HPV-positive patients with and without a smoking history. Cheng et al. (54) studied 70 patients with advanced OPSCC (13 patents HPV-positive and 57 patients HPV-negative), and the patients were followed up for at least 24 mo or until death. Multivariate Cox regression analysis showed that age, tumor total lesion glycolysis, and uniformity were independently associated with progression-free survival and disease-specific survival. Total lesion glycolysis, uniformity, and HPV positivity were significantly associated with overall survival.

\section{THERAPY RESPONSE ASSESSMENT}

Many studies have proposed that PET/CT imaging may improve the accuracy of posttreatment evaluation for HNSCC (57-61). It has been demonstrated that the metabolic response closely correlates with the histopathologic response, and survival is far better in responders than in nonresponders (Figs. 3 and 4). The timing of posttherapy PET/CT is crucial in the posttherapy assessment. The optimum timing after chemotherapy and radiotherapy is not known, but an interval of $12 \mathrm{wk}$ has been generally recommended to balance the drawbacks of imaging too early versus too late. Accuracy is generally greater for scans performed more than $12 \mathrm{wk}$ after radiation, likely because of a reduction in radiationinduced inflammation (62). Recently, we reported that the percentage of ${ }^{18}$ F-FDG PET/CT studies that are indeterminate because of possible treatment-related inflammation stabilizes between 4 and 24 mo after treatment and that the most appropriate timing for posttherapy PET/CT is between 3 and 4 mo (63). 


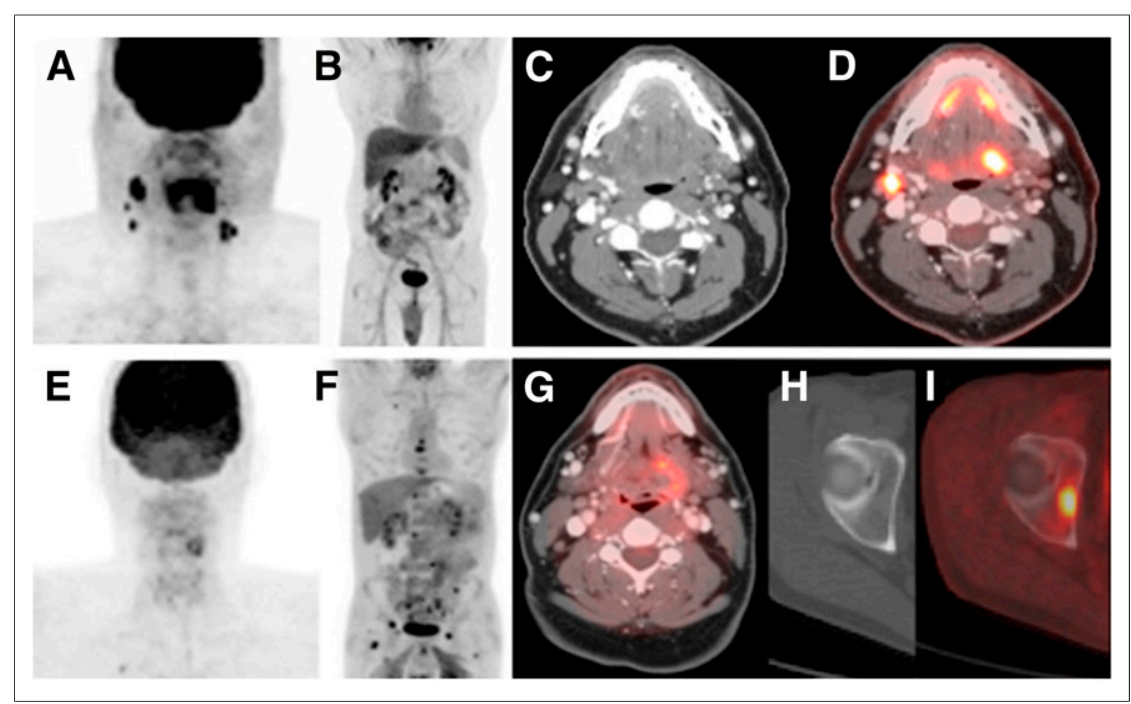

FIGURE 4. Disease recurrence with distant metastases in unusual sites (multiple skeletal) and disseminating phenotype in 46-y-old man with history of HPV-positive squamous cell carcinoma of left base of tongue, stage T1 N2c M0. Staging scan was performed using maximum-intensity projection of head and neck (A) and whole body (B), axial enhanced CT (C), and enhanced PET/

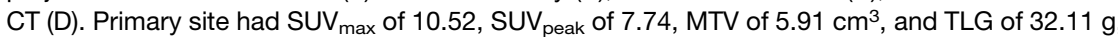
with locoregional nodal metastases bilaterally in neck. Treatment was with definitive radiation and cisplatin (200-cGy fractional dose with 35 fractions, for total dose of 70 Gy). Enhanced PET/CT 4 mo after end of treatment showed recurrent disease. Restaging scan with maximum-intensity projection of head and neck $(E)$ and whole body $(F)$ demonstrated significant progression, with recurrent disease in left base of tongue $(\mathrm{G})$ and multiple intense foci of ${ }^{18} \mathrm{~F}-\mathrm{FDG}$ uptake scattered throughout axial skeleton, including right acetabulum (I) with no obvious corresponding CT abnormalities $(\mathrm{H})$. HPV status may have greater influence on pattern than does delayed distant spread. Surfacing at distant sites rarely targeted by HPV-negative cancers may be related to repression of viral oncogene expression, which induces massive apoptosis and restoration of p53 and retinoblastoma protein tumor suppressor pathways.

Studies have shown that HPV-positive HNSCCs respond better to chemotherapy and radiation than HPV-negative ones $(29,64,65)$. In a retrospective study by Zhang et al. (64), the first posttreatment ${ }^{18} \mathrm{~F}$-FDG PET/CT scan was obtained between 4 and $16 \mathrm{wk}$ (median, $9 \mathrm{wk}$ ) after treatment in $62 \mathrm{HNSCC}$ patients. The median follow-up for disease-free patients was 21 mo from completion of treatment. Disease-free survival was associated with ${ }^{18}$ F-FDG PET/CT outcome (log-rank $\left.P<0.001\right)$ and HPV status (log-rank $P=0.01$ ), showing superior treatment response in HPVpositive HNSCC patients.

Studies demonstrating the value of PET/CT in management of the neck after radiotherapy with or without chemotherapy in HPVpositive OPSCC patients are limited and evolving (Table 1). Chan et al. (66) studied 77 patients with HPV-positive OPSCC who underwent contrast-enhanced PET/CT after radiotherapy as the primary treatment. The authors showed that enhanced PET/CT has a better negative predictive value than either enhanced CT or nonenhanced PET/CT alone in patients with HPV-positive OPSCC. Furthermore, they concluded that PET/CT with an SUV $\mathrm{Sax}_{\text {max }}$ threshold of 2 in patients with HPV-positive OPSCC offers a high negative predictive value $(100 \%)$ that may obviate unnecessary neck dissections. Moeller et al. (67) conducted a prospective study of 98 patients with HNSCC for posttherapy assessment. Only 1 of 3 patients had a known HPV status. The authors concluded that ${ }^{18} \mathrm{~F}$ -

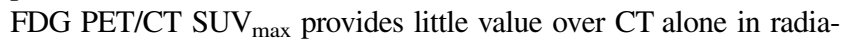
tion response assessment for unselected patients with locally advanced HNSCC. However, ${ }^{18}$ F-FDG PET/CT SUV $\max$ improved assessment of treatment response in high-risk patients, such as those with HPV-negative and nonoropharyngeal disease, and unlike the CT reads, the PET/CT reads were not dichotomized into positive and negative for residual tumor based on qualitative assessment. As the HPV-positive OPSCC clinical paradigms evolve, future studies are warranted to fully establish the value of ${ }^{18} \mathrm{~F}$ FDG PET/CT in assessing therapy for these patients.

\section{SUMMARY}

HPV-positive OPSCC represents an emerging disease that differs from HPVnegative OPSCC in natural history and prognosis. Contrast-enhanced PET/CT is essential to accurately stage the primary site when there are smaller tumors and to detect locoregional nodal metastases when there is a higher proportion of cystic nodal metastases and distant metastases manifesting in unusual sites with a disseminating phenotype. In addition, ${ }^{18} \mathrm{~F}-\mathrm{FDG}$ MTV is emerging as a prognostic parameter for outcome in HPV-positive OPSCC patients.

For these HPV-positive OPSCC patients, individualizing patient management is a real possibility, with reduction of radiotherapy dose to reduce treatment-related morbidities. ${ }^{18}$ F-FDG PET/CT has proven to be useful in assessing therapy for HNSCC, and early studies have shown similar efficacy for HPV-positive OPSCC. ${ }^{18}$ F-FDG PET/CT will also play a role in the therapy assessment of HPV-positive OPSCC patients in the setting of dose reduction, initially to prove these dosereduction strategies are efficacious without compromising patient outcome. The better prognosis and outcome of HPV-positive patients would likely warrant less intense imaging follow-up during the 5-y follow-up period after treatment. However, the manifestation of distant metastases later in the disease course and at unusual sites with a disseminating phenotype would require a longer follow-up with PET/CT. At this stage, it is unclear whether identifying distant metastases earlier during follow-up would improve patient outcome.

\section{REFERENCES}

1. Mehanna H, Paleri V, West CM, Nutting C. Head and neck cancer: part 1epidemiology, presentation, and prevention. BMJ. 2010;341:c4684.

2. Chaturvedi AK, Engels EA, Anderson WF, Gillison ML. Incidence trends for human papillomavirus-related and -unrelated oral squamous cell carcinomas in the United States. J Clin Oncol. 2008;26:612-619.

3. Chaturvedi AK, Engels EA, Pfeiffer RM, et al. Human papillomavirus and rising oropharyngeal cancer incidence in the United States. J Clin Oncol. 2011;29: 4294-4301.

4. Marur S, D’Souza G, Westra WH, Forastiere AA. HPV-associated head and neck cancer: a virus-related cancer epidemic. Lancet Oncol. 2010;11:781789.

5. Smith EM, Ritchie JM, Summersgill KF, et al. Human papillomavirus in oral exfoliated cells and risk of head and neck cancer. J Natl Cancer Inst. 2004;96: 449-455. 
6. D'Souza G, Kreimer AR, Viscidi R, et al. Case-control study of human papillomavirus and oropharyngeal cancer. N Engl J Med. 2007;356:1944-1956.

7. Sacks A, Peller PJ, Surasi DS, Chatburn L, Mercier G, Subramaniam RM. Value of PET/CT in the management of primary hepatobiliary tumors, part 2. AJR. 2011;197:W260-W265.

8. Subramaniam RM, Wilcox B, Aubry MC, Jett J, Peller PJ. ${ }^{18}$ F-fluoro-2-deoxy-Dglucose positron emission tomography and positron emission tomography/ computed tomography imaging of malignant pleural mesothelioma. $\mathrm{J} \mathrm{Med}$ Imaging Radiat Oncol. 2009;53:160-169.

9. Wilcox BE, Subramaniam RM, Peller PJ, et al. Utility of integrated computed tomography-positron emission tomography for selection of operable malignant pleural mesothelioma. Clin Lung Cancer. 2009;10:244-248.

10. Subramaniam RM, Clayton AC, Karantanis D, Collins DA. Hibernoma: ${ }^{18}$ F FDG PET/CT imaging. J Thorac Oncol. 2007;2:569-570.

11. Davison J, Mercier G, Russo G, Subramaniam RM. PET-based primary tumor volumetric parameters and survival of patients with non-small cell lung carcinoma. AJR. 2013;200:635-640.

12. Dibble EH, Karantanis D, Mercier G, Peller PJ, Kachnic LA, Subramaniam RM. PET/CT of cancer patients: part 1, pancreatic neoplasms. AJR. 2012;199: 952-967.

13. Davison JM, Ozonoff A, Imsande HM, Grillone GA, Subramaniam RM. Squamous cell carcinoma of the palatine tonsils: FDG standardized uptake value ratio as a biomarker to differentiate tonsillar carcinoma from physiologic uptake. Radiology. 2010;255:578-585.

14. Imsande HM, Davison JM, Truong MT, et al. Use of ${ }^{18} \mathrm{~F}$-FDG PET/CT as a predictive biomarker of outcome in patients with head-and-neck non-squamous cell carcinoma. AJR. 2011;197:976-980.

15. Dibble EH, Alvarez AC, Truong MT, Mercier G, Cook EF, Subramaniam RM. ${ }^{18}$ F-FDG metabolic tumor volume and total glycolytic activity of oral cavity and oropharyngeal squamous cell cancer: adding value to clinical staging. $\mathrm{J} \mathrm{Nucl}$ Med. 2012;53:709-715.

16. Jackson T, Chung MK, Mercier G, Ozonoff A, Subramaniam R. FDG PET/CT interobserver agreement in head and neck cancer: FDG and CT measurements of the primary tumor site. Nucl Med Commun. 2012;33:305-312.

17. Paidpally V, Chirindel A, Lam S, Agrawal N, Quon H, Subramaniam RM. FDGPET/CT imaging biomarkers in head and neck squamous cell carcinoma. Imaging Med. 2012;4:633-647.

18. Weinberger PM, Yu Z, Haffty BG, et al. Molecular classification identifies a subset of human papillomavirus-associated oropharyngeal cancers with favorable prognosis. J Clin Oncol. 2006;24:736-747.

19. Smeets SJ, Hesselink AT, Speel EJ, et al. A novel algorithm for reliable detection of human papillomavirus in paraffin embedded head and neck cancer specimen. Int J Cancer. 2007;121:2465-2472.

20. Licitra L, Perrone F, Bossi P, et al. High-risk human papillomavirus affects prognosis in patients with surgically treated oropharyngeal squamous cell carcinoma. J Clin Oncol. 2006;24:5630-5636.

21. Hoffmann M, Ihloff AS, Gorogh T, et al. p16(INK4a) overexpression predicts translational active human papillomavirus infection in tonsillar cancer. Int $J$ Cancer. 2010;127:1595-1602.

22. Pannone G, Rodolico V, Santoro A, et al. Evaluation of a combined triple method to detect causative HPV in oral and oropharyngeal squamous cell carcinomas: p16 immunohistochemistry, consensus PCR HPV-DNA, and in situ hybridization. Infect Agent Cancer. 2012;7:4.

23. Reimers N, Kasper HU, Weissenborn SJ, et al. Combined analysis of HPV-DNA, p16 and EGFR expression to predict prognosis in oropharyngeal cancer. Int $J$ Cancer. 2007;120:1731-1738.

24. Singhi AD, Westra WH. Comparison of human papillomavirus in situ hybridization and p16 immunohistochemistry in the detection of human papillomavirusassociated head and neck cancer based on a prospective clinical experience. Cancer. 2010;116:2166-2173.

25. Thomas J, Primeaux T. Is p16 immunohistochemistry a more cost-effective method for identification of human papilloma virus-associated head and neck squamous cell carcinoma? Ann Diagn Pathol. 2012;16:91-99.

26. El-Mofty SK, Lu DW. Prevalence of human papillomavirus type 16 DNA in squamous cell carcinoma of the palatine tonsil, and not the oral cavity, in young patients: a distinct clinicopathologic and molecular disease entity. Am J Surg Pathol. 2003;27:1463-1470.

27. Gillison ML, Koch WM, Capone RB, et al. Evidence for a causal association between human papillomavirus and a subset of head and neck cancers. J Natl Cancer Inst. 2000;92:709-720.

28. El-Mofty SK, Zhang MQ, Davila RM. Histologic identification of human papillomavirus (HPV)-related squamous cell carcinoma in cervical lymph nodes: a reliable predictor of the site of an occult head and neck primary carcinoma. Head Neck Pathol. 2008;2:163-168.
29. Fakhry C, Westra WH, Li S, et al. Improved survival of patients with human papillomavirus-positive head and neck squamous cell carcinoma in a prospective clinical trial. J Natl Cancer Inst. 2008;100:261-269.

30. Ang KK, Harris J, Wheeler R, et al. Human papillomavirus and survival of patients with oropharyngeal cancer. $N$ Engl J Med. 2010;363:24-35.

31. O'Rorke MA, Ellison MV, Murray LJ, Moran M, James J, Anderson LA. Human papillomavirus related head and neck cancer survival: a systematic review and meta-analysis. Oral Oncol. 2012;48:1191-1201.

32. Brix G, Lechel U, Glatting G, et al. Radiation exposure of patients undergoing whole-body dual-modality ${ }^{18} \mathrm{~F}-\mathrm{FDG}$ PET/CT examinations. J Nucl Med. 2005;46:608-613.

33. Huang B, Law MW, Khong PL. Whole-body PET/CT scanning: estimation of radiation dose and cancer risk. Radiology. 2009;251:166-174.

34. Murano T, Minamimoto R, Senda M, et al. Radiation exposure and risk-benefit analysis in cancer screening using FDG-PET: results of a Japanese nationwide survey. Ann Nucl Med. 2011;25:657-666.

35. Koch WM. Clinical features of HPV-related head and neck squamous cell carcinoma: presentation and work-up. Otolaryngol Clin North Am. 2012;45: 779-793.

36. Subramaniam RM, Truong M, Peller P, Sakai O, Mercier G. Fluorodeoxyglucose-positron-emission tomography imaging of head and neck squamous cell cancer. AJNR. 2010;31:598-604.

37. Krabbe CA, Balink H, Roodenburg JL, Dol J, de Visscher JG. Performance of ${ }^{18} \mathrm{~F}$-FDG PET/contrast-enhanced CT in the staging of squamous cell carcinoma of the oral cavity and oropharynx. Int J Oral Maxillofac Surg. 2011;40: 1263-1270.

38. Goldenberg D, Begum S, Westra WH, et al. Cystic lymph node metastasis in patients with head and neck cancer: an HPV-associated phenomenon. Head Neck. 2008;30:898-903.

39. Lassen P, Eriksen JG, Krogdahl A, et al. The influence of HPV-associated p16expression on accelerated fractionated radiotherapy in head and neck cancer: evaluation of the randomised DAHANCA 6\&7 trial. Radiother Oncol. 2011;100: 49-55.

40. Rischin D, Young RJ, Fisher R, et al. Prognostic significance of p16INK4A and human papillomavirus in patients with oropharyngeal cancer treated on TROG 02.02 phase III trial. $J$ Clin Oncol. 2010;28:4142-4148.

41. Haerle SK, Strobel K, Ahmad N, Soltermann A, Schmid DT, Stoeckli SJ. Contrast-enhanced ${ }^{18} \mathrm{~F}$-FDG-PET/CT for the assessment of necrotic lymph node metastases. Head Neck. 2011;33:324-329.

42. Huang SH, Perez-Ordonez B, Weinreb I, et al. Natural course of distant metastases following radiotherapy or chemoradiotherapy in HPV-related oropharyngeal cancer. Oral Oncol. 2013;49:79-85.

43. O'Sullivan B, Huang SH, Perez-Ordonez B, et al. Outcomes of HPV-related oropharyngeal cancer patients treated by radiotherapy alone using altered fractionation. Radiother Oncol. 2012;103:49-56.

44. Chan SC, Wang HM, Yen TC, et al. ${ }^{18}$ F-FDG PET/CT and 3.0-T whole-body MRI for the detection of distant metastases and second primary tumours in patients with untreated oropharyngeal/hypopharyngeal carcinoma: a comparative study. Eur J Nucl Med Mol Imaging. 2011;38:1607-1619.

45. Ng SH, Chan SC, Yen TC, et al. PET/CT and 3-T whole-body MRI in the detection of malignancy in treated oropharyngeal and hypopharyngeal carcinoma. Eur J Nucl Med Mol Imaging. 2011;38:996-1008.

46. Xu GZ, Zhu XD, Li MY. Accuracy of whole-body PET and PET-CT in initial M staging of head and neck cancer: a meta-analysis. Head Neck. 2011;33: 87-94.

47. Machtay M, Moughan J, Trotti A, et al. Factors associated with severe late toxicity after concurrent chemoradiation for locally advanced head and neck cancer: an RTOG analysis. J Clin Oncol. 2008;26:3582-3589.

48. Caudell JJ, Schaner PE, Meredith RF, et al. Factors associated with long-term dysphagia after definitive radiotherapy for locally advanced head-and-neck cancer. Int J Radiat Oncol Biol Phys. 2009;73:410-415.

49. Kies MS, Holsinger FC, Lee JJ, et al. Induction chemotherapy and cetuximab for locally advanced squamous cell carcinoma of the head and neck: results from a phase II prospective trial. J Clin Oncol. 2010;28:8-14.

50. Langendijk JA, Doornaert P, Verdonck-de Leeuw IM, Leemans CR, Aaronson NK, Slotman BJ. Impact of late treatment-related toxicity on quality of life among patients with head and neck cancer treated with radiotherapy. J Clin Oncol. 2008;26:3770-3776.

51. Quon H, Richmon JD. Treatment deintensification strategies for HPV-associated head and neck carcinomas. Otolaryngol Clin North Am. 2012;45: $845-861$.

52. Tang C, Murphy JD, Khong B, et al. Validation that metabolic tumor volume predicts outcome in head-and-neck cancer. Int J Radiat Oncol Biol Phys. 2012;83:1514-1520 
53. Cheng NM, Chang JT, Huang CG, et al. Prognostic value of pretreatment ${ }^{18} \mathrm{~F}-$ FDG PET/CT and human papillomavirus type 16 testing in locally advanced oropharyngeal squamous cell carcinoma. Eur J Nucl Med Mol Imaging. 2012;39: 1673-1684.

54. Cheng NM, Dean Fang YH, Tung-Chieh Chang J, et al. Textural features of pretreatment ${ }^{18} \mathrm{~F}$-FDG PET/CT images: prognostic significance in patients with advanced T-stage oropharyngeal squamous cell carcinoma. J Nucl Med. 2013;54: 1703-1709.

55. Joo YH, Yoo IR, Cho KJ, Park JO, Nam IC, Kim MS. Preoperative F-FDG PET/ $\mathrm{CT}$ and high-risk HPV in patients with oropharyngeal squamous cell carcinoma. Head Neck. June 1, 2013 [Epub ahead of print].

56. Tahari AK, Alluri K, Quon H, Koch W, Wahl R, Subramaniam R. FDG PET/CT imaging of oropharyngeal squamous cell carcinoma: characteristics of human papillomavirus-positive and -negative tumors. Clin Nucl Med. October 22, 2013 [Epub ahead of print].

57. Rabalais AG, Walvekar R, Nuss D, et al. Positron emission tomography-computed tomography surveillance for the node-positive neck after chemoradiotherapy. Laryngoscope. 2009;119:1120-1124.

58. Passero VA, Branstetter BF, Shuai Y, et al. Response assessment by combined PET-CT scan versus CT scan alone using RECIST in patients with locally advanced head and neck cancer treated with chemoradiotherapy. Ann Oncol. 2010;21:2278-2283.

59. Wang YF, Liu RS, Chu PY, et al. Positron emission tomography in surveillance of head and neck squamous cell carcinoma after definitive chemoradiotherapy. Head Neck. 2009;31:442-451.

60. Abgral R, Querellou S, Potard G, et al. Does ${ }^{18}$ F-FDG PET/CT improve the detection of posttreatment recurrence of head and neck squamous cell carcinoma in patients negative for disease on clinical follow-up? J Nucl Med. 2009;50: 24-29.

61. Yao M, Smith RB, Hoffman HT, et al. Clinical significance of postradiotherapy $\left[{ }^{18} \mathrm{~F}\right]$-fluorodeoxyglucose positron emission tomography imaging in management of head-and-neck cancer: a long-term outcome report. Int J Radiat Oncol Biol Phys. 2009;74:9-14.

62. Gupta T, Master Z, Kannan S, et al. Diagnostic performance of post-treatment FDG PET or FDG PET/CT imaging in head and neck cancer: a systematic review and meta-analysis. Eur J Nucl Med Mol Imaging. 2011;38:2083-2095.

63. Paidpally V, Tahari AK, Lam S, et al. Addition of ${ }^{18} \mathrm{~F}-\mathrm{FDG}$ PET/CT to clinical assessment predicts overall survival in HNSCC: a retrospective analysis with follow-up for 12 years. J Nucl Med. 2013;54:2039-2045.

64. Zhang I, Branstetter BF, Beswick DM, Maxwell JH, Gooding WE, Ferris RL. The benefit of early PET/CT surveillance in HPV-associated head and neck squamous cell carcinoma. Arch Otolaryngol Head Neck Surg. 2011;137: 1106-1111.

65. Mellin Dahlstrand H, Lindquist D, Bjornestal L, et al. P16(INK4a) correlates to human papillomavirus presence, response to radiotherapy and clinical outcome in tonsillar carcinoma. Anticancer Res. 2005;25(6C):4375-4383.

66. Chan JY, Sanguineti G, Richmon JD, et al. Retrospective review of positron emission tomography with contrast-enhanced computed tomography in the posttreatment setting in human papillomavirus-associated oropharyngeal carcinoma. Arch Otolaryngol Head Neck Surg. 2012;138:1040-1046.

67. Moeller BJ, Rana V, Cannon BA, et al. Prospective risk-adjusted $\left[{ }^{18} \mathrm{~F}\right]$ fluorodeoxyglucose positron emission tomography and computed tomography assessment of radiation response in head and neck cancer. J Clin Oncol. 2009;27: 2509-2515. 